\title{
A Utilização Da Capacidade Dinâmica De Inovação E A Gestão Estratégica De Pessoas, Como Preditores Ao Desempenho na Indústria Hoteleira
}

The Use Of Dynamic Innovation Capability And Strategic Human Resource Management as Predictors To The Hotel Industry Performance

Deosir Flávio Lobo de Castro Júnior (deosir@ifsc.edu.br)-Instituto Federal de Santa Catarina IFSC, Brazil

Marcio N. Miura (adm.miura@gmail.com)- Universidade Estadual do Oeste do Paraná (UNIOESTE), Brazil

Dr. Elvis Silveira-Martins (elvis.professor@gmail.com) - Universidade Federal de Pelotas - UFPel, Brazil

Pesquisador Jonatas Ramos (jonatas.rrafael@gmail.com) - Instituto Federal de Santa Catarina IFSC, Brazil

\section{RESUMO}

A presente investigação possui o objetivo de estudar a relação de predição ao melhor desempenho na indústria hoteleira da Grande Florianópolis. Para tanto, buscou-se o conhecimento científico sobre capacidade dinâmica de inovação, e gestão estratégica de pessoas e verificou-se a sua relação com o desempenho superior. No que tange aos procedimentos metodológicos, a abordagem define-se como quantitativo exploratório. Quanto a sua natureza é uma pesquisa aplicada. A população a ser pesquisa, encontram-se os hotéis executivos na Grande Florianópolis. Os respondentes, obrigatoriamente, ocupavam cargos de gestão, nos hotéis pesquisados, coletandose 141 questionários. Classifica-se como não-probabilística, e por conveniência. Os procedimentos para análise dos dados, utilizouse as análises fatoriais exploratórios, análises fatoriais confirmatórias, e modelagem de equações estruturais. Por meio 
das análises dos dados pode-se afirmar que existe a relação direta, positiva e significante para a capacidade dinâmica de inovação, na dimensão inovação de serviços e desempenho, assim como gestão estratégica de pessoas com o desempenho hoteleiro. No efeito indireto, Gestão estratégica de pessoas mediam a relação entre as duas dimensões de Capacidade Dinâmica de Inovação, de inovação gerencial e inovação de serviços, e o desempenho hoteleiro. Para futuras pesquisas sugere-se que a capacidade dinâmica mercadológica pode ser inserida neste modelo.

Palavras-chave: Capacidade Dinâmica Inovação, Gestão Estratégica de Pessoas, Desempenho Hoteleiro.

\section{ABSTRACT}

The present investigation aims to study the prediction relation for the best performance in the hotel industry of Greater Florianópolis. In order to do so, we sought scientific knowledge about dynamic capacity for innovation, and strategic management of people and verified their relationship with superior performance. Regarding methodological procedures, the approach is defined as exploratory quantitative. As far as its nature is an applied research. The population to be surveyed are executive hotels in Greater Florianópolis. The respondents, necessarily, held management positions in the hotels surveyed, collecting 141 questionnaires. It is classified as non-probabilistic, for convenience. The procedures for data analysis included exploratory factor analysis, confirmatory factor analysis, and modeling of structural equations. Through the analysis of the data, it can be affirmed that there is a direct, positive and significant relationship for the dynamic capacity of innovation, in the innovation dimension of services and performance, as well as strategic management of people with hotel performance. In the indirect effect, Strategic People Management measures the relationship between the two dimensions of Dynamic Capacity of Innovation, managerial innovation and service innovation, and hotel performance. For future research it is suggested that the dynamic marketing capacity can be inserted in this model.

Keywords: Dynamic Capacity Innovation; Strategic Management of People; Hotel Performance.

\section{INTRODUÇÃO}

O turismo, segundo a Organização Mundial do Turismo (2017) é definitivamente uma importante atividade econômica que por si só contribui 
com 10\% do Produto interno Bruto (PIB) em todo o mundo, em efeito direto, indireto e induzido de 1 em cada 11 dos postos de trabalhos. De acordo como Ministério do Turismo (2017, p. 4), "a Indústria hoteleira é responsável por 350 mil empregos formais e 1,5 milhões de ocupações indiretas em todo o Brasil". Denotando a sua importância, dentro do setor turístico, social e econômico.

O turismo, obrigatoriamente é o lugar para a inovação. O motivo desta assertiva pragmática inicia-se na multiplicidade de cenários competitivos, que contextualizam o ambiente onde se encontram as organizações, assim como os destinos turísticos. No setor turístico, a inovação em serviços apresenta-se como fundamental para se manter competitivo, e as necessárias adequações às tendências e exigências de seu mercado. Verificou-se a constante atenção a inovação, como um desafio na rotina organizacional, que procuram ofertar novos serviços para manter-se no mercado turístico, compreendido como ambiente competitivo. Ressalta-se que nos processos de inovação, apresenta-se não apenas uma intenção de mudar, mas, especialmente, um esforço integrado, em ações coordenados entre o conhecimento e a ação. É necessário reconhecer e admitir as tendências e as demandas da sociedade e do seu mercado-alvo, assim como entregar a estrutura e os recursos essenciais ao desenvolvimento da inovação (Machado, Dreher, \& Gorni, 2009; Souza, Pena, \& Moesch, 2017).

Como justificativa, para a presente investigação, concluem Araújo e Ernesto Filho (2013), que os meios de hospedagem precisam aperfeiçoar no âmbito tecnológico, assim como no aspecto mercadológico. Destacando as dimensões da inovação, a capacitação dos gestores no que tange os aspectos da inovação. Aires (2017) afirma ainda, que inovação em hotéis é uma situação sine qua non para o desenvolvimento nas atividades turísticas. Destacando a escassez de pesquisas, que abordam, objetivam e contemplam esta temática. Na concepção de inovação da investigação, ficou evidenciado o conceito, a importância e a implementação de inovações. Aponta a autora, que os gestores devem investir no 
A UTILIZAÇÃO DA CAPACIDADE DINÂMICA DE INOVAÇÃO E A GESTÃO ESTRATÉGICA DE PESSOAS,

COMO PREDITORES AODESEMPENHO NA INDÚSTRIA HOTELEIRA

desenvolvimento da melhoria de serviços, a partir das sugestões dos funcionários e parcerias junto aos hóspedes.

Deluca, Gonçalo, Castro e Pereira (2017) bem como Tometich, Fracasso e Zen (2014), reconhecem uma diversidade de abordagens no que se refere as capacidades dinâmicas, estabelecendo uma relação ao processo de gestão da inovação nas organizações. Desta forma, destacam na literatura científica, "capacidade" como sendo uma habilidade humana (Penrose, 1959; Barney, 1991); a existência de habilidades, experiências vivenciadas e conhecimentos presentes na empresa (Richardson, 1972). Tais visões confluem na mesma orientação, ao descrever a probabilidade das firmas em desenvolverem suas operações com maior competitividade. Concluem que as capacidades possibilitam que as empresas se desenvolvam em um ambiente dinâmico, ressaltando-se as capacidades dinâmicas.

Castro, Deluca, Silveira-Martins, Miura e Martins (2016) afirmam que a capacidade dinâmica de inovação, é um tema internacionalmente relevante, discutida em pesquisas focadas em estratégias organizacionais. Os resultados demonstraram uma perspectiva global e a falta de pesquisas sobre o tema, e suas interrelações, como preditores ou mediando as relações com o desempenho. Os autores sugerem a realização de pesquisas com novas relações e o construto capacidade dinâmica de inovação.

Irving e Fragelli (2012) em sua pesquisa sobre a inovação e planejamento turístico, verificaram o valor do elemento humano, neste processo. Castro, Gonçalo e Rossetto (2014), concluíram que o elemento humano, está relacionado com o melhor desempenho na hotelaria, e media a relação entre as capacidades dinâmicas mercadológica e o desempenho superior, na indústria hoteleira catarinense.

Em um ambiente dinâmico e multifacetado, as redes de hotéis, os complexos turísticos e as empresas que oferecem serviços de alimentos e bebidas tiveram de aumentar as práticas de desenvolvimento e capacitação de pessoal para cargos operacionais e gerenciais (Wood, 
1995). Nesse caminho, Tomasia (2000) relata que, para se sobreviver e manter competitiva, a indústria hoteleira necessita, de um conjunto de funcionários bem treinados, assim como de gerentes estratégicos e intermediários adequadamente capacitados e competentes na área de gestão de pessoas e talentos, de maneira estruturada e ativa.

Na hotelaria, a hospedagem é o principal produto intangível e a qualidade é percebida pelo cliente por meio de suas experiências e expectativas, quanto ao serviço prestado. Os colaboradores são, portanto, os responsáveis por atender as expectativas dos clientes e os gestores por sua vez, tem a tarefa complexa de motivá-los (Castro et al., 2014).

Dessa forma, a gestão de pessoas para hotelaria requer muita atenção, pois além de gerirem as atividades de rotina dos setores, devem proporcionar um bom clima de trabalho, diminuir o turnover, incentivar atitudes positivas, motivar os funcionários para que estes estejam aptos para prestar um serviço de qualidade (Petrocchi, 2002, Barreto, Albuquerque, \& Medeiros, 2014, Santos \& Estender, 2016).

Na busca de melhorar o desempenho hoteleiro, e na manutenção da sua competitividade entre os meios de hospedagem, a hotelaria busca inovar em seus serviços e nos seus processos de gestão, bem como, investir na melhor capacitação da gestão de pessoas. Diante do exposto, pergunta-se, qual a relação da capacidade dinâmica inovativa e da gestão estratégica de pessoas vem contribuindo no desempenho superior dos hotéis da Grande Florianópolis?

Esta pesquisa tem por objetivo verificar a relação da capacidade dinâmica de inovação, assim como a gestão estratégica de pessoas, como preditor de um desempenho superior, na hotelaria executiva e econômica na grande Florianópolis.

\section{REFERENCIAL TEÓRICO}

\section{Capacidade dinâmica de inovação}


Costa e Nunez (2016) definem inovação como sendo o processo que determina aquilo que possui a percepção do novo, abarcando as alterações, modificações, modernizações, novas configurações, destacam a concordância e aproveitamento pela sociedade.

Schumpeter (1997) categorizou as inovações em: i) inovações radicais, denominada as que produzem fortes interrupções, ii) inovações incrementais, destinadas as mudanças que possuem alguma continuidade. Podendo ser: i) Relacionadas a novos serviços e produtos; ii) Nova forma de processo produtivo; iii) desenvolvimento de mercados inexistentes; iv) invenções ou reconhecimentos fontes alternativas de insumos e matériasprimas, e v) desenvolvimento de novas configurações de mercados e serviços industriais.

Por oportuno, destaca-se que a capacidade de inovação em ambiente mutante, é reconhecido como capacidade dinâmica de inovação, por relacionar-se em como a empresa desenvolve seus processos produtivos, de forma proativa ou orientada à inovação (Menguc \& Auh, 2006).

Chadha (2009) conclui que a capacidade dinâmica de inovação é percebida, em sendo o processo que auxilia ao desenvolvimento de novas mercadorias, peças, objetos, artefatos, elementos ou processos. Em observância a concorrência, identifica-se que os ciclos de vida dos produtos estão menores, com inserção de novas gerações cada vez em período menos, o que valoriza a pesquisa e desenvolvimento de novos serviços/produtos, o que dificulta a imitação das empresas inovadoras, diferenciando sua oferta e melhorando a competitividade a médio e longo prazo. Shoham, Vigoda-Gadot, Ruvio e Schwabsky (2012) dividem em cinco dimensões, a inovação em ambiente dinâmico e mutável, conforme se apresenta no quadro 1. 


\begin{tabular}{|c|c|}
\hline Dimensão & \\
\hline Criatividade & $\begin{array}{l}\text { Implementação de novas ideias de produtos, } \\
\text { serviços, processos, etc. }\end{array}$ \\
\hline Assunção ao Risco & $\begin{array}{l}\text { Direcionamento de recursos em tomadas de } \\
\text { decisão }\end{array}$ \\
\hline Orientação Futura & $\begin{array}{l}\text { Possibilita de maneira mais prática a adaptação da } \\
\text { organização em mercados de rápida mudança }\end{array}$ \\
\hline Abertura a Mudanças & Condição da organização em adotar inovações \\
\hline Proatividade & $\begin{array}{l}\text { Quando a organização consegue antecipar-se às } \\
\text { mudanças que ocorrem no mercado e assim, } \\
\text { aproveita oportunidades potenciais }\end{array}$ \\
\hline
\end{tabular}

Quadro 1 - Dimensões da Inovação

Fonte: Adaptado de Shoham et al. (2012)

Deluca et al. (2017), Yesil, Koska e Büyükbese (2013) e Liao, Wu, Hu e Tsuei (2009) analisaram a relação entre a capacidade dinâmica de inovação e o desempenho organizacional, e concluíram em suas investigações que as inovações em serviços e produtos, resultam em desempenho superiores.

O modelo apresentado por Liao, Fei e Chen (2007), testados e validados na realidade organizacional brasileira (Escobar, 2012, Deluca et al., 2017) confirmam que a capacidade dinâmica da inovação, auxiliam as empreas a alcançarem a desejada vantagem entre seus competidores, em mercados complexos e dinâmicos, com a inovação e a flexibilidade estratégica. Desenvolvendo os aspectos técnicos da inovação e, os aspectos de gestão da inovação (Figura 1).

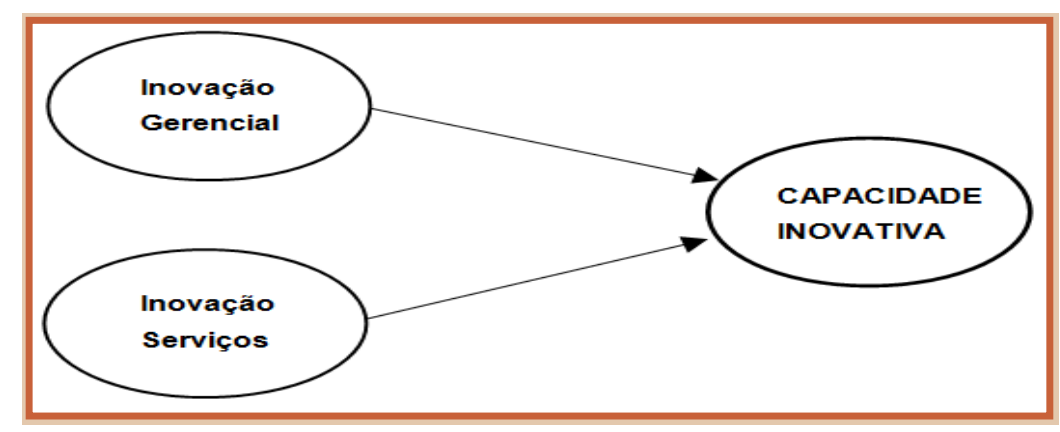

Figura 1 - Modelo Capacidade Inovativa de Liao, Fei e Chen

Fonte: Liao, Fei e Chen (2007). 
Desta forma, assume-se que a capacidae inovativa é formada a partir de duas dimensões, a inovação gerencial e a inovação de serviços. Sendo assim utilizada na modelagem teórica e teórica-prática.

\section{Gestão estratégica de pessoas na hotelaria}

A gestão de pessoas é um processo que envolve planejamento, liderança, organização, direção e controle as atividades de pessoas, com desempenho eficiente para alcançar os mesmos objetivos da organização (Vilas Boas \& Andrade, 2009).

Neste contexto, na administração hoteleira, estão inseridas as práticas de gestão de pessoas que, com as rápidas mudanças ambientais, principalmente econômicas e tecnológicas chegaram a patamares estratégicos. O conjunto destas práticas, bem-sucedidas objetiva incrementar a competitividade aos meios de hospedagem, assim como, de agregar valor ao produto/serviço (Camargos \& Dias, 2010, Ferreira, 2013).

A gestão estratégica, no contexto organizacional, é um método escolhido intencionalmente pela organização, para que seus objetivos organizacionais sejam alcançados, a longo prazo. Estando, desta forma, ligada ao planejamento, ao investimento e ao desenvolvimento das capacidades, e das competências de seus colaboradores (Pimentel, 2016, Silva \& Souza, 2017).

Estudos apontam para a adoção, pelas organizações, das práticas da gestão estratégica de pessoas. Todavia, a decisão deve ser definida alinhada a política estratégica da organização. Na área da hotelaria é de fundamental importância o relacionamento entre os diversos setores e seus agentes dentro da organização. Estas relações em sua maioria são dependentes do comportamento humano. Assim, podemos observar a relação direta entre a gestão de pessoas e o desempenho organizacional (Budhwar \& Aryee, 2008; Pimentel, 2016, Echeverria, Crepaldi, \& Bianchi, 2017). 
Para se obter qualidade desejável, nos serviços prestados, se torna fundamental manter uma equipe envolvida, e capacitada, seja para o planejamento ou para a execução dos processos da organização. Portanto, a oferta de treinamentos, e da capacitação de seus colaboradores, apresenta-se como uma maneira a apresentar um diferencial na hotelaria, conseguindo a obtenção da vantagem competitiva (Gonçalves \& Bahia, 2011; Caldas \& Martins, 2017).

Echeverria, Crepaldi e Bianchi (2017), concluem que é por meio dos treinamentos, e da capacitação, que se desenvolve e se dissemina o conhecimento organizacional, e se melhora as habilidades. Para que seja possível aperfeiçoar as habilidades, e capacitar os colaboradores, se faz necessário também um planejamento adequado. Iniciar pelo diagnóstico da necessidade de capacitação, a elaboração de um plano de ação voltada ao treinamento, a implementação de forma consistente, e conduzido com eficácia. Por fim, com a avaliação desse processo o mesmo poderá ser validado, readequado ou descartado.

A organização que prioriza e reconhece a importância da gestão de pessoas, aliada às estratégias organizacionais, possui uma vantagem competitiva e um diferencial perante as demais (Freitas, 2012). As organizações hoteleiras, em que as atividades de gestão de pessoas estejam alinhadas às estratégias organizacionais tem por consequência uma visão mais ampla das necessidades de seus colaboradores e são capazes de oferecer benefícios que complementem a remuneração (Nichele, Stefano e Raifur, 2015).

A gestão estratégica de pessoas na indústria hoteleira é especialmente importante ao passo que selecionar pessoas adequadas, treinar e desenvolvê-las, de modo a retê-las na organização colabora para um serviço de qualidade como resultado a satisfação e fidelização do cliente. (Pimentel, 2016).

Pelo supra apresentado, surgem as três hipóteses do presente estudo: 
Ho - Não há relação entre a Capacidade Dinâmica de Inovação, Gestão Estratégica de Pessoas com o Desempenho hoteleiro e nem mediação nesta relação.

H1 (a; b) - Capacidade Dinâmica de Inovação possui relação positiva e significante com o Desempenho Hoteleiro.

H2 - Gestão Estratégica de Pessoas possui relação positiva e significante com 0 Desempenho Hoteleiro.

H3 (a; b) - Gestão Estratégica de Pessoas media a relação entre a Capacidade Dinâmica da Inovação e o Desempenho Hoteleiro.

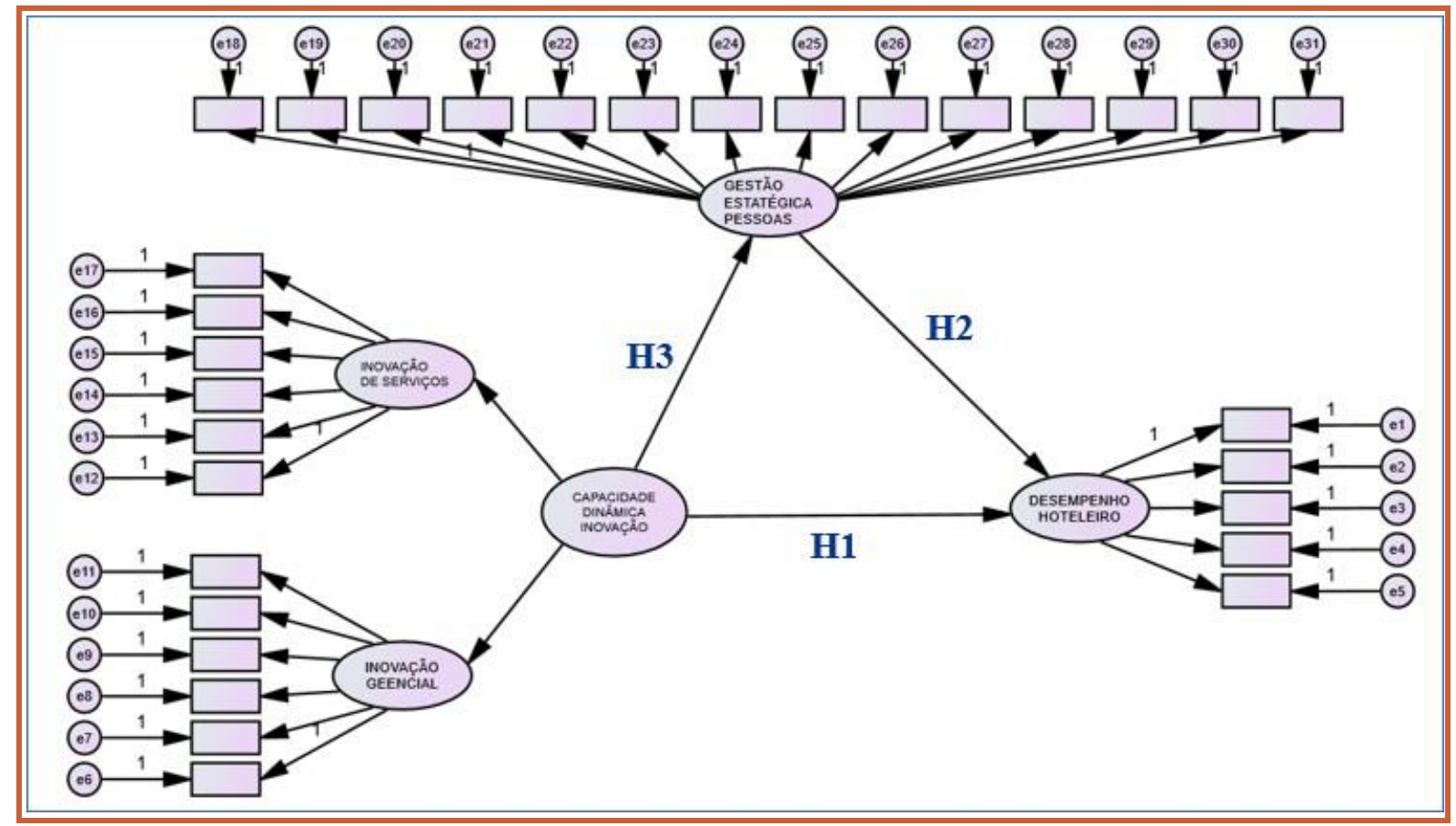

Figura 2 - Modelo teórico

Fonte: Dados da Pesquisa

\section{METODOLOGIA}


O presente estudo é definido, em sua abordagem, como sendo quantitativo exploratório, o que permite maior conhecimento do problema a ser estudado. Para complementar a pesquisa exploratória, foi utilizada pesquisa descritiva, objetivando contribuir e possibilitar a descrição de fenômenos, assim como comparar e avaliar o que o objeto de estudo. $O$ objeto de estudo foram os hotéis executivos e econômicos, localizados na região da Grande Florianópolis.

A presente pesquisa, quanto a sua natureza é uma pesquisa aplicada. Quanto a lógica filosófica, recorreu-se ao hipotético-dedutivo. Em relação ao objetivo do estudo, caráter exploratório, descritivo. Para a coleta dos dados, por se tratar de uma pesquisa quantitativa, utilizou-se a técnica Survey para se coletar os dados.

Quanto a população pesquisada, seu universo encontram-se todos os gerentes de hotéis executivos e econômicos na grande Florianópolis. Os respondentes desta investigação, obrigatoriamente, eram ocupantes de cargos de gestão, nos hotéis pesquisados. A amostra, classifica-se como nãoprobabilística, e por conveniência, sendo coletados 141 questionários validados.

No que se refere aos procedimentos para análise dos dados, utilizou-se as análises fatoriais exploratórios, análises fatoriais confirmatórias e modelagem de equações estruturais. A elaboração do instrumento de coleta de dados, recorreu-se a escalas validadas em pesquisa anteriores, com fundamentação teórica consistente. Os questionários continham perguntas fechadas, com escala tipo Likert de 7 pontos. Nos quadros 2, 3 e 4, apresentam-se as variáveis e medidas, utilizadas no presente estudo.

Capacidade dinâmica de Inovação - Dimensão Inovação Gerencial

IG1 - A equipe de gestão possui participação estratégica, ou seja, ela está totalmente engajada, dispensando horas na definição de estratégia e da maneira de pô-la em prática;

IG2 - A equipe de gestão possui uma compreensão muito boa dos detalhes das operações do negócio;

IG3 -Há uma melhoria da capacidade de recrutar e reter profissionais altamente qualificados e com uma elevada capacidade de inovação e criatividade; 
Capacidade dinâmica de Inovação - Dimensão Inovação Gerencial

IG4 - Há um sistema de incentivos e de bem estar pessoal;

IG5 - Há uma busca constante por novas maneiras sem que se possam equilibrar as atividades em diferentes áreas do negócio;

Capacidade dinâmica de Inovação - Dimensão Inovação Serviços

IS1 - Há inovação incremental de serviço, ou seja, capacidade de desenvolver mudanças que incrementam os serviços e produzam melhoras;

I\$2 - Há inovação radical de serviços, ou seja, capacidade de desenvolver novos serviços;

IS3 - Há diferenciação comercial do serviço, ou seja, capacidade de diferenciar uma gama de serviços comercialmente;

IS4 - Há conhecimento diversificado do serviço, ou seja, capacidade de desenvolver conhecimento aplicado para a criação uma carteira diversificada de serviço;

IS5 - Há adaptabilidade do serviço, ou seja, capacidade de ajustar o design do serviço às necessidades do cliente e responder rápida e eficazmente às mudanças dessas necessidades;

Quadro 2 - Variáveis e Medidas - Construto Capacidade de Inovação

Fonte: Liao, Fei e Chen (2007).

\section{Desempenho Hoteleiro}

DH1 - Vendas totais

DH2 - Taxa média de ocupação

DH3 - Margem de lucro sobre as vendas totais

DH4 - Diária média

DH5 - Vendas por unidade habitacional

DH6 - Custo médio por diária vendida

Quadro 3 - Variáveis e Medidas - Construto Desempenho

Fonte: Carvalho (2011).

\begin{tabular}{|l|}
\hline Gestão Estratégica de Pessoas \\
\hline GP 1 - Investir em educação, treinamento e desenvolvimento de pessoas \\
\hline GP 2 - Promover um melhor ambiente de trabalho \\
\hline GP 3 - Favorecer o alcance das metas organizacionais \\
\hline GP 4 - Investir em programas de retenção de talentos \\
\hline GP 5 - Promover uma administração eficiente \\
\hline GP 6 - Estimular o comprometimento das pessoas com a empresa \\
\hline GP 7 - Disseminar e fortalecer a cultura organizacional \\
\hline GP 8 - Incentivar a criatividade na busca de melhorias contínuas \\
\hline GP 9 - Investir no desenvolvimento de gestores e lideranças \\
\hline GP10 - Estimular o desenvolvimento e gestão de carreiras \\
\hline GP11 - Promover a gestão de pessoas com foco nos resultados \\
\hline GP12 - Promover a aprendizagem organizacional \\
\hline GP13 - Alcançar a excelência operacional nos serviços de gestão de pessoas \\
\hline GP14 - Incentivar e praticar a responsabilidade social e ambiental \\
\hline GP15 - Estimular a melhoria contínua dos processos organizacionais \\
\hline GP16 - Desenvolver competências organizacionais e individuais \\
\hline GP17 - Atrair funcionários competentes \\
\hline GP18 - Desenvolver uma cultura de alta performance \\
\hline
\end{tabular}




\begin{tabular}{|l|}
\hline GP19 - Estimular a integração dos funcionários e o trabalho em equipe \\
\hline GP20 - Investir no controle e na padronização de procedimentos \\
\hline GP21 - Promover a remuneração variável de longo prazo \\
\hline GP22 - Desenvolver comunicação transparente e eficaz \\
\hline GP23 - Preparar a organização para enfrentar os desafios futuros \\
\hline GP24 - Implantar ou reestruturar processos de remuneração e de benefícios \\
\hline GP25 - Identificar e implantar melhores práticas de gestão de pessoas \\
\hline GP26 - Facilitar a transformação organizacional \\
\hline GP27 - Reconhecer e recompensar pessoas \\
\hline GP28 - Estimular a delegação e a autonomia (empoderamento) \\
\hline GP29 - Promover a diversidade e a inclusão \\
\hline GP30 - Estimular a gestão do conhecimento \\
\hline GP31 - Estimular e apoiar as iniciativas dos funcionários \\
\hline GP32 - Investir no desenvolvimento de novas tecnologias e métodos de trabalho
\end{tabular}

Quadro 4 - Variáveis e Medidas - Construto Gestão Estratégica de Pessoas

Fonte: Barreto, Albuquerque, \& Medeiros (2014)

\section{ANÁLISE DOS DADOS}

No que se refere as análises dos dados, foram seguidos os parâmetros estatísticos, cientificamente validados, para a análise fatorial exploratória, com os testes a serem submetidos e valores mínimos no quadro 1.

A análise descritiva, e a verificação da unidimensionalidade foram alcançadas por meio da utilização do software SPSS $\circledast$, versão 24.0. Utilizou-se o método de extração, por meio da análise de componentes principais.

\begin{tabular}{|l|l|}
\hline \multicolumn{1}{|c|}{ MEDIDAS } & \multicolumn{2}{c|}{ VALORES MÍNIMOS ESPERADOS } \\
\hline Comunalidades & 0,50 \\
\hline Carga fatorial & $\mathbf{0 , 7 0}$ \\
\hline Medida de adequação da amostra (MSA) & 0,50 \\
\hline KMO & $\mathbf{0 , 5 0}$ \\
\hline Teste de esfericidade de Barlett & $\mathrm{P}<0,05$ \\
\hline Alfa de Cronbach & $\mathbf{0 , 7 0}$ \\
\hline Correlação inter-itens & $\mathbf{0 , 3 0}$ \\
\hline Correlação itens-total & $\mathbf{0 , 5 0}$ \\
\hline
\end{tabular}

Quadro 5 - Medidas e Valores Esperados AFE

Fonte: Adaptado de Hair, Black, Babin, \& Anderson (2010)

Sendo aprovadas as variáveis de medidas para o construto Capacidade Dinâmica de Inovação, IS2; IS3, IS4 e IS5, na dimensão inovação de serviços e as variáveis de medidas IGI; IG2; IG3 e IG4; na dimensão inovação de gerencial. Construto bidimensional.

No que tange as variáveis de medidas aprovadas para o construto Gestão Estratégica de Pessoas, as variáveis GP2, GP3; GP5; GP6; GP7; GP8; 
GP9; GP11; GP12; GP13 e GP19. Construto unidimensional, aprovado estatisticamente.

Para o construto Desempenho Hoteleiro, as variáveis de medidas aprovadas foram $\mathrm{DH} 1$; DH2; DH3; $\mathrm{DH} 4$ e DH5.

Após a realização dos testes, da análise fatorial exploratória, quadro 5, as variáveis deste estudo foram submetidas a análise fatorial confirmatória, assim como aos ajustes para o modelo a conceitual ser testado, os testes a serem submetidos e valores mínimos constam no quadro 6.

\begin{tabular}{|c|c|}
\hline MEDIDAS & VALORES MÍNIMOS ESPERADOS \\
\hline$X^{2}\left(Q u i^{2}\right)$ & $P>a$ \\
\hline $\mathrm{X}^{2}$ / Graus de Liberdade (Qui'/GL) & $<3,000$ \\
\hline $\mathbf{P}$ & $>0,050$ \\
\hline RMSEA & $<0,100$ \\
\hline CFI & $>0,900$ \\
\hline TLI & $>0,900$ \\
\hline NFI & $>0,900$ \\
\hline
\end{tabular}

Quadro 6 - Resultados esperados para a AFC e ajustes do Modelo de Equações Estruturais Fonte: Adaptado de Hair et al. (2010)

\section{Análise fatorial confirmatória - AFC}

As variáveis foram submetidas aos testes de: Comunalidades; Carga fatorial (para $\mathrm{n}$ até 200); KMO; Teste de esfericidade de Bartlett; Alfa de Cronbach; Correlação inter-itens e Correlação item-total. As variáveis aprovadas, em todos nestes testes, foram submetidas aos testes da análise fatorial confirmatória.

AFC - Construto Gestão de Pessoas

Após os testes da análise fatorial confirmatória, a modelagem do construto, figura 3 , e seus resultados são apresentados na tabela 1. Sendo aprovado o construto Gestão Estratégica de Pessoas. 


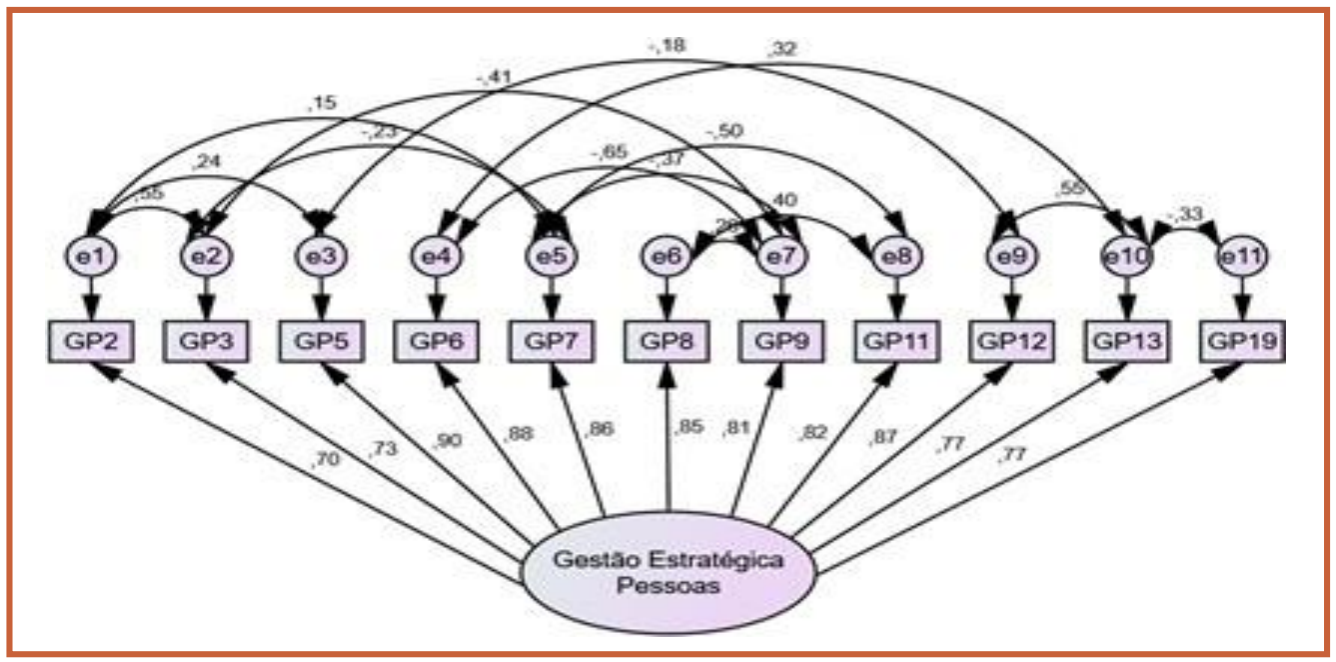

Figura 3 - AFC - Construto Gestão Estratégica de Pessoas

Fonte: Dados da Pesquisa

Abaixo, os resultados Análise Fatorial Confirmatória do Gestão de Pessoas, unidimensional, dos testes de Chi quadrado, Chi quadrado dividido pelos graus de liberdades, significância, erro quadrático, que estão dentro dos valores esperados, sendo aceitos estatisticamente. Os testes de ajustes CFI, TLI e NFI também se ajustaram perfeitamente. Construto aprovado

\begin{tabular}{|c|c|c|}
\hline Índice & Valores Modelo Final & Valores Esperados \\
\hline$X^{2}$ & $\mathbf{3 3 , 8 3 7}$ & \\
\hline$X^{2} / \mathrm{GL}$ & $\mathbf{1 , 1 2 8}(33,837 / 30)$ & $>3,000$ \\
\hline $\mathrm{P}$ & $\mathbf{0 , 2 8 7}$ & $<0,100$ \\
\hline $\mathrm{RMSEA}$ & $\mathbf{0 , 0 5 6}$ & $>0,900$ \\
\hline $\mathrm{CFI}$ & $\mathbf{0 , 9 9 2}$ & $>0,900$ \\
\hline $\mathrm{TLI}$ & $\mathbf{0 , 9 8 5}$ & $>0,900$ \\
\hline $\mathrm{NFI}$ & $\mathbf{0 , 9 3 5}$ & \\
\hline
\end{tabular}

Tabela 1 - Índices de ajuste do Gestão Estratégica de PessoasFonte:

Dados da Pesquisa

Tendo o construto Gestão Estratégica de Pessoas (GEP), sendo validado estatisticamente, passou o construto capacidade dinâmica de inovação, em suas duas dimensões aos testes da análise fatorial confirmatória. 
AFC - Construto Capacidade Dinâmica de Inovação - Inovação de Serviços

O construto capacidade dinâmica de inovação, foi inicialmente testada a dimensão inovação de serviços, conforme figura 4, e seus resultados apresentados na tabela 2.

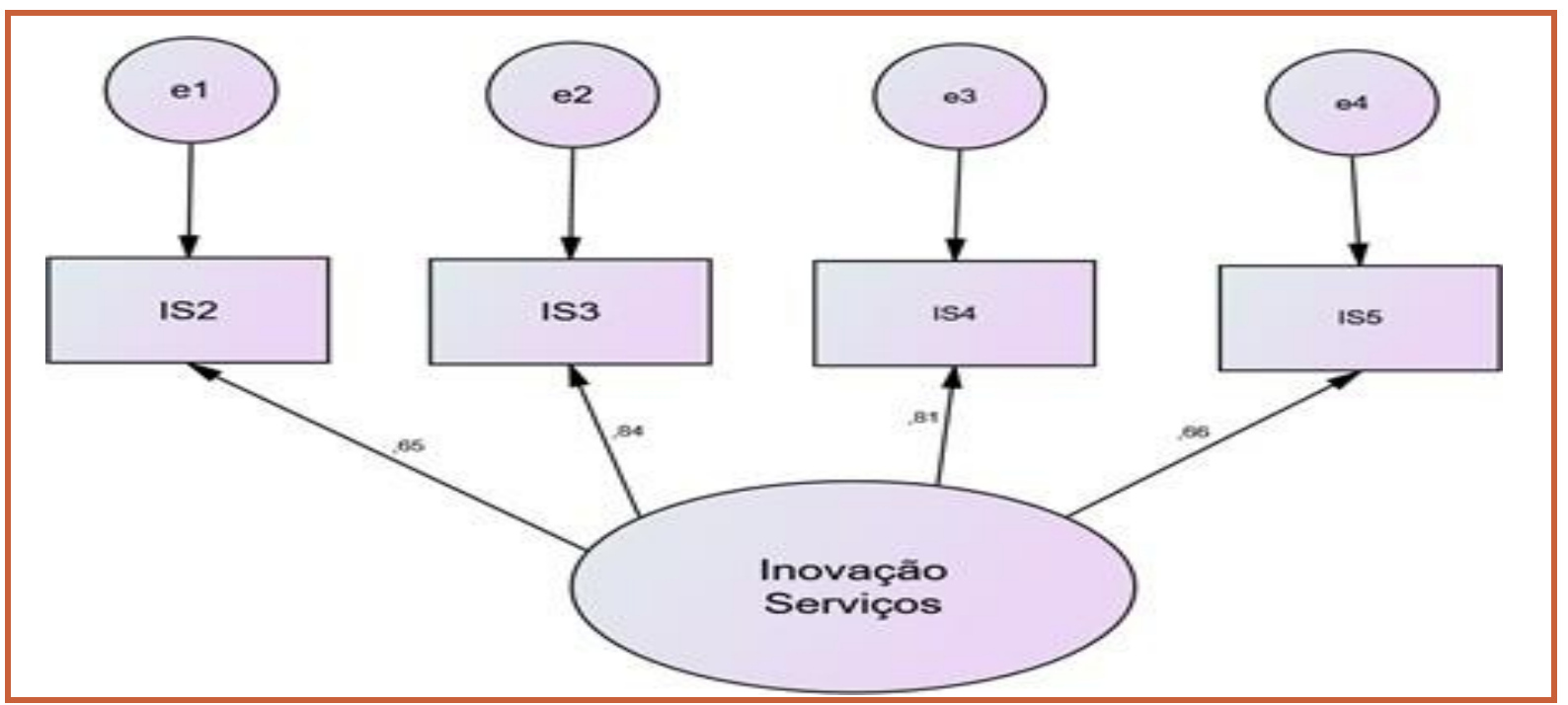

Figura 4 - AFC - Construto Capacidade Dinâmica de Inovação - Inovação de Serviços

Fonte: Dados da Pesquisa

Os resultados da Análise Fatorial Confirmatória, para o construto Desempenho Hoteleiro, unidimensional, passaram pelos testes de Chi quadrado, Chi quadrado dividido pelos graus de liberdades, significância, erro quadrático, que estão dentro dos valores esperados, sendo validados estatisticamente. Os testes de ajustes CFl, TLI e NFI também se ajustaram perfeitamente. Construto aprovado capacidade de inovação, na dimensão inovação de serviços. Resultados apresentados na tabela 2.

\begin{tabular}{|c|c|c|}
\hline İndice & Valores Modelo Final & Valores Esperados \\
\hline$X^{2}$ & $\mathbf{2 , 0 2 0}$ & $<3,000$ \\
\hline$X^{2} / G L$ & $\mathbf{1 , 0 1 0}(2,020 / 2)$ & $>0,050$ \\
\hline $\mathrm{P}$ & $\mathbf{0 , 3 6 4}$ & $<0,100$ \\
\hline $\mathrm{NMSEA}$ & $\mathbf{0 , 0 1 6}$ & $>0,900$ \\
\hline $\mathrm{CFI}$ & $\mathbf{0 , 9 9 9}$ & $>0,900$ \\
\hline $\mathrm{TLI}$ & $\mathbf{0 , 9 9 8}$ & $>0,900$ \\
\hline $\mathrm{NFI}$ & $\mathbf{0 , 9 4 5}$ & \\
\hline
\end{tabular}

Tabela 2 - Índices de ajuste - Capacidade Dinâmica de Inovação - Inovação de Serviços 
Fonte: Dados da Pesquisa

AFC - Construto Capacidade Dinâmica de Inovação - Inovação de Gerencial

O construto capacidade dinâmica de inovação, foi modelado na dimensão inovação gerencial, tendo sua modelagem representada na figura 5 , e os resultados obtidos na tabela 3.

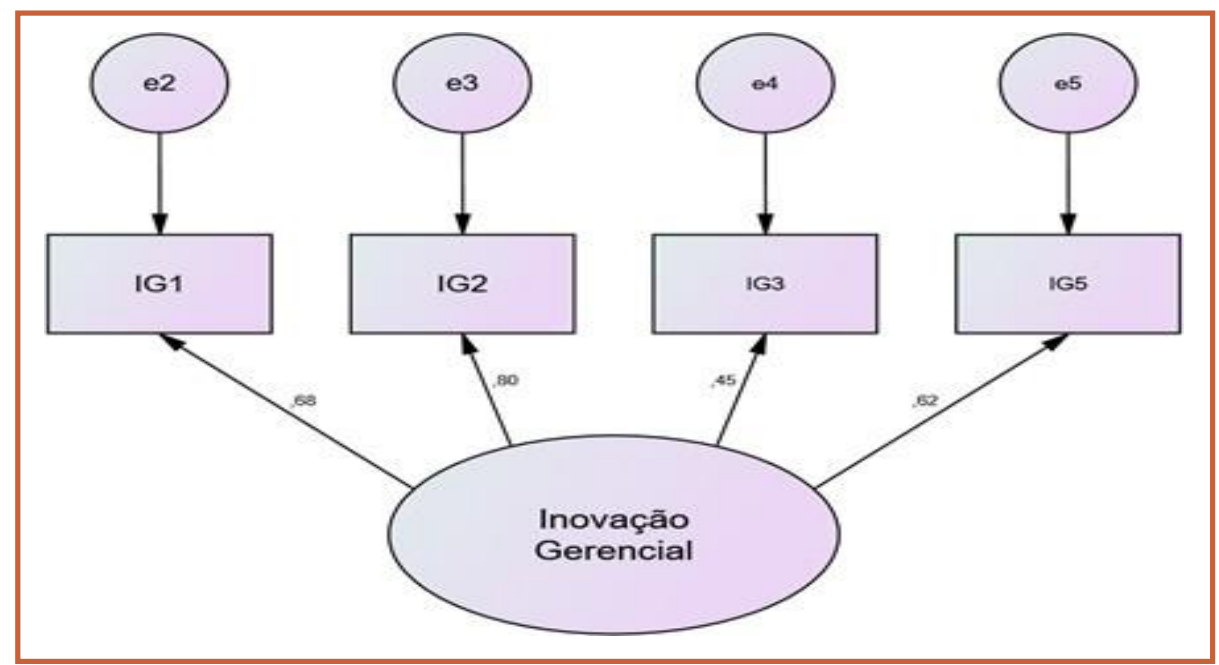

Figura 5 - AFC - Construto Capacidade Dinâmica de Inovação - Inovação Gerencial Fonte: Dados da Pesquisa

Abaixo, os resultados Análise Fatorial Confirmatória do Desempenho Hoteleiro, unidimensional, dos testes de Chi quadrado, Chi quadrado dividido pelos graus de liberdades, significância, erro quadrático, que estão dentro dos valores esperados, sendo aceitos estatisticamente. Os testes de ajustes CF, TLl e NFl também se ajustaram perfeitamente. Construto aprovado.

\begin{tabular}{|c|c|c|}
\hline Índice & Valores Modelo Final & Valores Esperados \\
\hline$X^{2}$ & $\mathbf{2 , 6 0 6}$ & $<3,000$ \\
\hline$X^{2} / G L$ & $\mathbf{1 , 3 0 3}(2,606 / 2)$ & $>0,050$ \\
\hline $\mathrm{P}$ & $\mathbf{0 , 2 7 2}$ & $<0,100$ \\
\hline $\mathrm{RMSEA}$ & $\mathbf{0 , 0 8 6}$ & $>0,900$ \\
\hline $\mathrm{CFl}$ & $\mathbf{0 , 9 9 0}$ & $>0,900$ \\
\hline $\mathrm{TLI}$ & $\mathbf{0 , 9 6 9}$ & $>0,900$ \\
\hline $\mathrm{NFI}$ & $\mathbf{0 , 9 5 9}$ & \\
\hline
\end{tabular}

Tabela 3 - Índices de ajuste - Capacidade Dinâmica de Inovação - Inovação de Gerencial 
Fonte: Dados da Pesquisa

Após a validação do construto Capacidade Dinâmica de Inovação, em suas duas dimensões (Inovação de Serviços e Inovação Gerencial), realizou-se a análise fatorial confirmatória do construto Desempenho Hoteleiro (DH).

AFC - Construto Desempenho Hoteleiro

Foi testado a modelagem do construto Desempenho Hoteleiro, Figura 6, e seus resultados apurados são apresentados na Tabela 4.

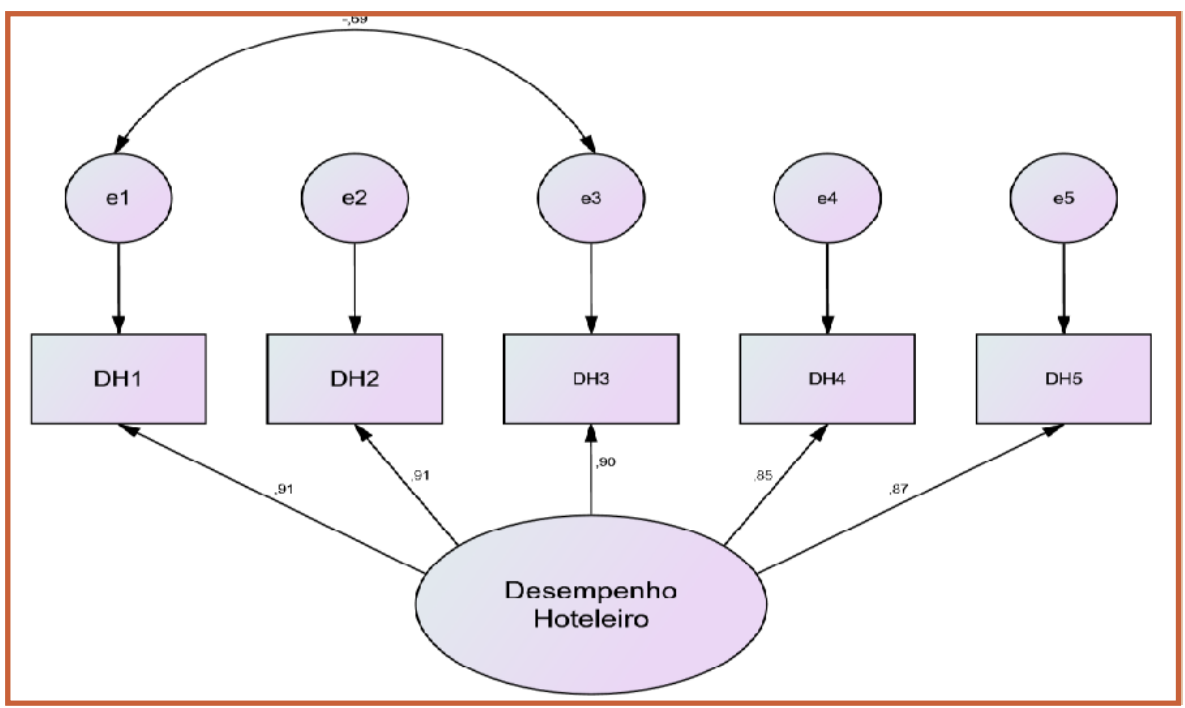

Figura 6 - AFC - Desempenho Hoteleiro

Fonte: Dados da Pesquisa

Na tabela 4 são apresentados os resultados Análise Fatorial Confirmatória do Desempenho Hoteleiro, unidimensional. Sendo aprovados pelos testes de Chi quadrado, Chi quadrado dividido pelos graus de liberdades, significância, erro quadrático, que estão dentro dos valores esperados, sendo aceitos estatisticamente. Os testes de ajustes CFI, TLI e NFI também se ajustaram perfeitamente. Aprovando o Construto Desempenho Hoteleiro. 


\begin{tabular}{|c|c|c|}
\hline Índice & Valores Modelo Final & Valores Esperados \\
\hline$X^{2}$ & $\mathbf{8 , 7 5 0}$ & \\
\hline$X^{2} / G L$ & $\mathbf{2 , 1 8 8}(8,750 / 4)$ & $<3,000$ \\
\hline$P$ & $\mathbf{0 , 0 6 8}$ & $>0,050$ \\
\hline RMSEA & $\mathbf{0 , 0 9 0}$ & $<0,100$ \\
\hline CFI & $\mathbf{0 , 9 7 6}$ & $>0,900$ \\
\hline TLI & $\mathbf{0 , 9 3 9}$ & $>0,900$ \\
\hline NFI & $\mathbf{0 , 9 5 8}$ & $>0,900$ \\
\hline
\end{tabular}

Tabela 4 - Índices de ajuste - Desempenho HoteleiroFonte:

Dados da Pesquisa

Uma vez que cada uns dos quatro (4) construtos se comportaram favoravelmente, unidimensionais, aprovados nos testes da análise fatorial exploratória e pela análise fatorial confirmatória, passou a etapa final, aos testes de modelagem de equações estruturais.

\section{Modelo teórico-empírico}

O modelo teórico-empírico é apresentado sua representação gráfica na Figura 7, com suas cargas de relação, e as covariâncias necessárias parao ajuste do modelo testado. Na tabela 5 são apresentados os resultados, indicies de ajustes do modelo geral e sua mensuração. 


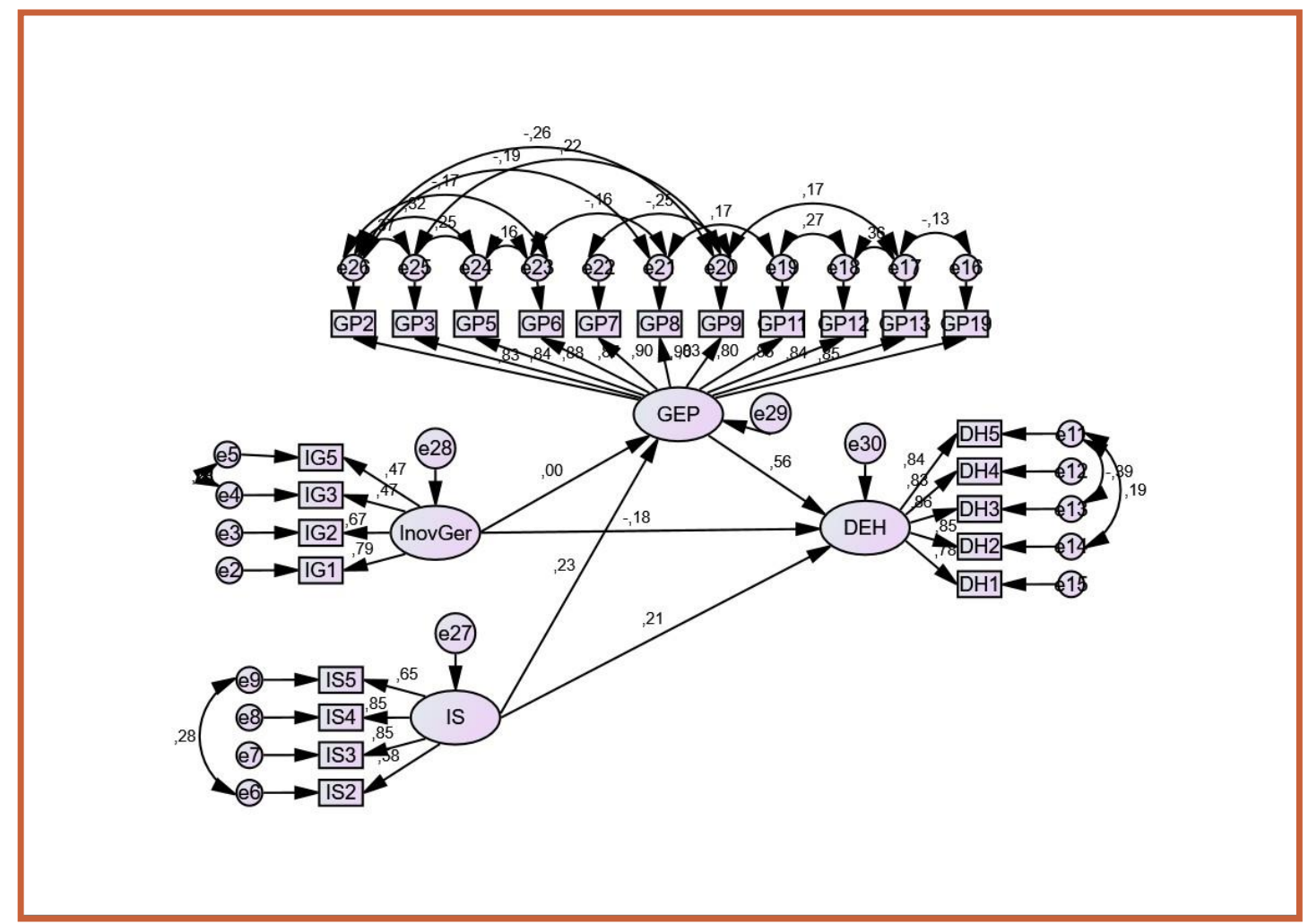

Figura 7 - Modelagem de Equações Estruturais

Fonte: Dados da pesquisa

Abaixo, os resultados Análise Fatorial Confirmatória do Gestão de Pessoas, unidimensional, dos testes de Chi quadrado, Chi quadrado dividido pelos graus de liberdades, significância, erro quadrático, que estão dentro dos valores esperados, sendo aceitos estatisticamente. Os testes de ajustes CFl, TLl e NFI também se ajustaram perfeitamente. Construto aprovado

\begin{tabular}{|c|c|c|}
\hline Índice & Valores Modelo Final & Valores Esperados \\
\hline$X^{2}$ & $\mathbf{3 2 7 , 9 8 0}$ & $<3,000$ \\
\hline$X^{2} / G L$ & $\mathbf{1 , 4 3 9}$ & $<0,050$ \\
\hline$P$ &, 000 & $<0,100$ \\
\hline RMSEA &, 056 & $>0,900$ \\
\hline CFI &, 962 & $>0,900$ \\
\hline TLI &, 953 & $>0,900$ \\
\hline NFI &, 886 & \\
\hline
\end{tabular}

Tabela 5 - Índices de ajuste do modelo geral de mensuraçãofonte:

Dados da Pesquisa 


\section{Testes de Hipóteses}

A partir dos resultados dos testes das hipóteses, do presente estudo, pode-se afirmar que a hipótese, $\mathrm{Hl}$, foi parcialmente suportada, confirmando em parte a teoria pesquisada. Pelos comportamentos dos dados coletados, pode-se concluir que existe efeito direto entre a Capacidade Dinâmica de Inovação, dimensão inovação de serviços, e o desempenho hoteleiros. A dimensão inovação gerencial não apresentou significância estatística, não suportando a hipótese.

A hipótese, H2, foi suportada, uma vez que a gestão estratégica de pessoas possui relação positiva e significante com o desempenho hoteleiro.

A hipótese, H3, também foi suportada em suas duas dimensões (inovação gerencial e inovação de serviços) tem a relação com o desempenho, mediado pelo construto gestão estratégica de pessoas.

Portanto é possível concluir, e afirmar, que a capacidade dinâmica de inovação é preditor da gestão estratégica de pessoas e do desempenho hoteleiro. Conforme os dados apresentados na Tabela 6.

\begin{tabular}{|c|c|c|c|c|c|}
\hline CAMINHO ESTRUTURAL. DIRETO & HIPÓTESE & $P$ & $\begin{array}{r}\text { COEFICIENTE } \\
\text { PADRONIZADO }\end{array}$ & RELAÇĀO & SUPORTE \\
\hline $\begin{array}{l}\text { Desempenho } \square \text { Capacidade Dinâmica } \\
\text { delnovação (Inovação Serviços) }\end{array}$ & $\mathrm{Hla}$ & 0,003 & 210 & Positiva & Suportada \\
\hline $\begin{array}{l}\text { Desempenho } \square \text { Capacidade dinâmica } \\
\text { de inovação (Inovação Gerencial) }\end{array}$ & $\mathrm{HIb}$ & 0,473 &,- 179 & Negativa & $\begin{array}{l}\text { Não } \\
\text { suportada }\end{array}$ \\
\hline $\begin{array}{l}\text { Desempenho } \square \text { Gestão Estratégica } \\
\text { Pessoas }\end{array}$ & $\mathrm{H} 2$ & 0,000 &, 556 & Positiva & Suportada \\
\hline CAMINHO ESTRUTURAL INDIRETO & HIPÓTESE & $P$ & $\begin{array}{r}\text { COEFICIENTE } \\
\text { PADRONIZADO } \\
\end{array}$ & RELAÇĀO & SUPORTE \\
\hline $\begin{array}{l}\text { Gestão Estratégica de Pessoas } \\
\text { Capacidade de } \\
\text { novação (Inovaçãoserviços) }\end{array}$ & $\mathrm{H} 3 \mathrm{a}$ & 1,000 & 0,000 & Media & Suportada \\
\hline $\begin{array}{l}\text { Gestão Estratégica de Pessoas } \\
\text { Capacidade dinâmica de inovação } \\
\text { (Inovação Gerencial) }\end{array}$ & $\mathrm{H} 3 \mathrm{~b}$ & 1,000 & 0,000 & Media & Suportada \\
\hline
\end{tabular}

Tabela 6 - Resumo das relações hipotéticas testadas

Fonte: Dados da Pesquisa

\section{CONCLUSÃO}


A presente pesquisa teve por objetivo verificar a relação de predição na hotelaria com o desempenho. Reuniu conhecimentos científicos que possibilitaram construir um modelo conceitual no qual a capacidade dinâmica de inovação e gestão estratégica de pessoas implica no desempenho hoteleiro. E dessa forma, contribuir com estudos sobre inovação e gestão de pessoas na região da grande Florianópolis.

Inicialmente foi refutada a hipótese H0, que afirmava que não há relação entre a Capacidade Dinâmica de Inovação, Gestão Estratégica de Pessoas com o Desempenho hoteleiro e nem mediação nesta relação. Pelas análises dos dados da presente investigação, pode-se afirmar que existem as relações entre a Capacidade Dinâmica de Inovação, Gestão Estratégica de Pessoas com o Desempenho hoteleiro, assim como a mediação nesta relação, confirmando as pesquisas de Escobar, Lizote, e Verdinelli (2012) eCastro Júnior et al (2020).

A hipótese Hla foi suportada. Identificou-se que existe uma relação positiva entre a Capacidade Dinâmica de Inovação possui relação positiva e significante com o Desempenho Hoteleiro, dimensão inovação de serviços $(0,210)$, significante $a a=0,03(p=0,000<a=0,05)$. Para a área da gestão nahotelaria, Machado (2009) afirma que o setor turístico se encontra cada vez mais competitivo e em constante inovação. Dessa forma, Escobar (2012) e Deluca et al. (2017) argumentam que a capacidade dinâmica de inovação colabora para o alcance de vantagem entre seus competidores.

Contudo a hipótese Hib não foi suportada. Não obstante a constatação da existência de uma relação negativa entre Capacidade Dinâmica de Inovação possui relação positiva e significante com o Desempenho Hoteleiro, na dimensão inovação gerencial $(-0,179)$, não sendo significante $a \mathrm{a}=0,05(\mathrm{p}=0,799>a=0,05)$.

A hipótese H2 foi suportada. Identificou-se que existe uma relação positiva entre a Gestão Estratégica de Pessoas possui relação positiva e significante com o Desempenho Hoteleiro $(0,556)$, significante a $a=0,00$ $(p=0,000<a=0,05)$. A gestão de pessoas alinhada às estratégias 
organizacionais tem papel importante para o desempenho do colaborador e qualidade dos serviços prestados pelo mesmo. Conforme as variáveis e medidas da pesquisa, GPl (investir em educação, treinamento e desenvolvimento) e GP8 (incentivar a criatividade na busca de melhorias contínuas) apontaram como principal influenciador do desempenho hoteleiro, confirmando as conclusões de Machado et al (2019).

A hipótese H3 foi suportada. Identificou-se que Gestão Estratégica de Pessoas media a relação entre a Capacidade Dinâmica da Inovação, em suas duas dimensões e o Desempenho Hoteleiro. Uma vez que toda a cargafoi absorvida no teste.

Pode-se observar que a hotelaria como prestadora de serviço requer uma equipe preparada e qualificada para atender as expectativas do hóspede e alcançar os objetivos organizacionais. Por meio da pesquisa, GP2 (um bom ambiente de trabalho), GP19 (integração da equipe) e GP11 (foco nos resultados) contribui para o desempenho das vendas do hotel. A habilidade de se adaptar às mudanças e inovar os serviços que atendem as necessidades do cliente (IS5) favorece a margem de lucro sobre as vendas totais. Confirmando as conclusões de Machado et al (2019) e Castro Júnior etal (2020).

Com a base teórica referido neste trabalho e da pesquisa realizada, junto aos hotéis executivos da grande Florianópolis, conta-se que o objetivo do trabalho foi absolutamente alcançado, tendo em vista que foi possível construir um modelo estrutural, que suporta a hipótese de haver relação entre as práticas de gestão de pessoas e capacidade dinâmica da inovação com o desempenho hoteleiro.

\section{REFERÊNCIAS}

Aires, J. D. M. (2017). A inovação na perspectiva de diretores de hotéis em Aveiro - Portugal. Revista Turismo - Visão e Ação, 19(3). 
Araújo, R. M., \& Ernesto, I, Fo. (2013). Meios de hospedagem: um estudo sobre marcas no contexto da inovação. Revista Iberoamericana de Turismo - RITUR, $3(1), 69-84$.

Barney, J. B. (1991). Firm resource and sustained competitive advantage. Journal of Management, 17(1), 99-120.

Barreto, L. M. T. S., Albuquerque, L. G., \& Medeiros, C. A. F. (2014). Estratégias de gestão de pessoas e desempenho organizacional na hotelaria: o papeldas capacidades organizacionais. Revista Brasileira de Pesquisa em Turismo RBTUR, 8(2), 340-360.

Budhwar, P., \& Aryee, S. (2008). An introduction to strategic human resource management, in the Aston Centre for Human Resources, Strategic Human Resource Management, Building Research-Based Practice, London: PICD.

Caldas, J. N., \& Martins, C. M. M. A. (2017). Gestão de pessoas no setor hoteleiro: um estudo de caso. Revista de Administração e Negócios da Amazônia, 9(4), 141-163.

Camargos, M. A., \& Dias, A. T. (2010). Estratégia, administração estratégica e estratégia corporativa: uma síntese teórica. REGE Revista de Gestão, 10(1), 2739.

Castro Junior, D. F. L.; Silveira-Martins, E.; Miura, M. N.; Abreu, J. C. (2020). Analysis of marketing capabilities, service differentiation and performance of executive hotels in the State of Santa Catarina, Brazil. INTERNATIONAL JOURNAL OF BUSINESS EXCELLENCE (ONLINE), 20, 451-470.

Castro, D. F. L. Jr., Deluca, M. A. M., Silveira-Martins, E., Miura, M. N., \& Martins, A. A. M. (2016). Innovation capacity and service differentiation: Strategies for competitive advantage, study methodologies. WSEAS Transactions on Business and Economics, 3, 1-10. 
Castro, D. F. L. Jr., Gonçalo, R. C., Rossetto, C. R., \& Deluca, M. A. M. (2016). Estudo dos reflexos das capacidades mercadológicas no desempenho da hotelaria executiva em Santa Catarina. Faces: Revista de Administração. $15(1), 44-63$.

Castro, D. F. L. Jr., Gonçalo, R. C., \& Rossetto, C. R. (2014). Diferenciação de serviços: construção e validação de escalas. Faces: Revista de Administração, 13, 46-64.

Chadha, A. (2009). Product cycles, innovation, and exports: a study of indian pharmaceuticals. World Development, 37(9), 1478-1483.

Costa, R. C., \& Nunez, C. V. (2016). Mercado de bioprodutos fitoterápicos e fitocosméticos: gestão, tecnologias e inovação. Revista Fitos, 10(3), 220- 372.

Deluca, M. A. M., Gonçalo, C. R., Castro, D. F. L. Jr., \& Pereira, M. F. (2017). Capacidades de inovação em serviços: um estudo nos supermercados em Santa Catarina. Revista lbero-Americana de Estratégia - RIAE, 16(1).

Echeverria, E. L., Crepaldi, M. L. S., \& Bianchi, E. (2017). O recrutamento, seleção e treinamento e seu impacto no desempenho da função na linha de produção. Revista Faipe, 3(1), 7-15.

Escobar, M. A. R., Lizote, S. A., \& Verdinelli, M. A. (2012). Relação entre orientação empreendedora, capacidade de inovação e munificência ambiental em agências de viagens. Turismo: Visão e Ação, 14(2), 269-286.

Freitas, J. C. T. (2012). Estratégia organizacional: utilizando a política de treinamento e desenvolvimento como vantagem competitiva. Anais do Simpósio de Excelência em Gestão Tecnologia - SEGET. Niterói, RJ, Brasil, 9.

Gonçalves, C. A. M. M., \& Bahia, L. R. G. (2011). Gestão Hoteleira. Manaus: Cetam, E-Tec Brasil. 
Hair, J. Jr., Black, W. C., Babin, B. J., \& Anderson, R. E. (2010). Multivariate data analysis. 7th ed. Upper Saddle River: Prentice Hall.

Irving, M. A., \& Fragelli, C. (2012). Turismo inclusivo: conceito vazio ou oportunidade de inovação em planejamento turístico? Revista Turismo \& Desenvolvimento, 3(7/18), 1431-1440.

Liao, S. H., Fei, W. C., \& Chen, C. C. (2007). Knowledge sharing, absorptive capacity and innovation capability: an empirical study of Taiwan"s knowledge intensive industries. Journal of Information Science, 33(1), 340-359.

Liao, S. H., Wu, C. C., Hu, D. C., \& Tsuei, G. A. (2009). Knowledge acquisition, absorptive capacity, and innovation capability: an empirical study of Taiwan's knowledge-intensive industries. International scholarly and Scientific Research \& Innovation, 3(5), 338-345.

Machado, D. D. P. N., Dreher, M. T., \& Gorni, P. M. (2009). Inovação em serviços turísticos: a percepção desse processo em agências de viagens. Observatório de Inovação do Turismo. 4(1).

Machado, L. A. N. ; Castro Júnior, Deosir Flávio Lobo De ; Mondo, T. S ; Silveira- Martins, E.; Abreu, J. C. (2019) . STRATEGIC HUMAN RESOURCES MANAGEMENT AND HOTEL PERFORMANCE. TOURISM \& MANAGEMENT STUDIES, 15, 65-79.

Menguc, B., \& Auh, S. (2006). Creating a firm-level dynamic capability through capitalizing on market orientation and innovativeness. Journal of the Academy of Marketing Science, 34(1), 63-73.

Ministério do Turismo - MTUR (2017). Marcos Conceituais. Brasília: MTUR.

Nichele, J., Stefano, S. R., \& Raifur, L. (2015). Análise da remuneração estratégica para atrair e reter colaboradores: a visão dos pós-graduandos. Revista de Carreiras e Pessoas. 5(2), 212-231. 
Organização Mundial do Turismo - OMT (2017). Why tourism. 2017. Madrid: OMT.

Penrose, E. (1959). The theory of the growth of the firm. London: Oxford University Press.

Petrocchi, M. (2002). Hotelaria: Planejamento e gestão. São Paulo: Futura.

Pimentel, H. (2016). O papel da gestão de pessoas no turismo. Revista Lusófona de Economia e Gestão das Organizações, 4, 81-111.

Richardson, G. B. (1972). The organization of industry. Economic Journal. 82, 883896.

Santos, J. A., \& Estender, A. C. (2016). Qualidade de vida e o impacto na rotatividade no setor hoteleiro. Revista FATEC Guarulhos: Gestão, Tecnologia \& Inovação. 2(1), 82-95.

Schumpeter, J. A. (1997). A teoria do desenvolvimento econômico: uma investigação sobre lucros, capital, juros e o ciclo econômico. São Paulo: Nova Cultural.

Shoham, A., Vigoda-Gadot, E., Ruvio, A. A., \& Schwabsky, N. (2012). Testing an organizational innovativeness integrative model across cultures, Journal of Engineering and Technology Management, 29(2), 226-240.

Silva, G. A., \& Souza, L. A. S. (2017). O impacto causado pelo clima organizacional no desempenho e na qualidade de vida da equipe de trabalho do Hotel Hardman - João Pessoa, PB. Revista Campo do Saber. $3(1)$.

Souza, L. H., Pena, L. C. S., \& Moesch, M. M. (2017). Conhecimento e sinergia como indutores da inovação regional em turismo: o caso do Observatório do Turismo no Distrito Federal (Brasil). Revista Brasileira de Pesquisa em Turismo RBTUR. 11(1), 19-38. 
Tomasia, M. T. (2000). Como motivar trabalhadores no ambiente empresarial segundo as teorias motivacionais. Londrina: FELCICAM/INBRAPE.

Tometich, P., Fracasso, E. M., \& Zen, A. C. (2014). Reconfiguring the firm's capabilities for innovation. Proceedings of the International Management of Technology Anval Conference-Science, Technology and Innovation in the age of Economic, Political and Security Challenges. Washington, DC, United States of America, 23.

Vilas Boas, A. A., \& Andrade, R. O. B. (2009). Gestão estratégica de pessoas. São Paulo: Elsevier.

Wood, T. Jr. (1995). Mudança organizacional: aprofundamento temas atuais em administração de empresas. São Paulo: Atlas.

Yesil, S., Koska, A., \& Büyükbese, T. (2013). Knowledge sharing process, innovation capability and innovation performance: an empirical study. Procedia Social and Behavioral Sciences, 75, 217-225. 